\title{
Strukturuntersuchungen an den Produkten aus der Reaktion des Tris( trimethylsilyl) silyllithiums mit Aceton
}

\author{
H. Oehme und R. Wustrack \\ Fachbereich Chemie der Universität Rostock, Buchbinderstraße 9, O-2500 Rostock (Deutschland)
}

\author{
A. Heine, G.M. Sheldrick und D. Stalke \\ Institut für Anorganische Chemie der Universität Göttingen, Tammannstraße 4, W-3400 Göttingen (Deutschland) \\ (Eingegangen den 7. Oktober 1992)
}

\begin{abstract}
The reaction of $\left(\mathrm{Me}_{3} \mathrm{Si}\right)_{3} \mathrm{SiLi}(1)$ with acetone has been clarified with the aid of crystal structure and ${ }^{29} \mathrm{Si}$ NMR investigations of $\mathrm{H}\left(\mathrm{Me}_{3} \mathrm{Si}\right)_{2} \mathrm{Si}-\left(\mathrm{Me}_{3} \mathrm{Si}\right)_{2} \mathrm{Si}-\mathrm{CMe}_{2} \mathrm{SiMe}_{3}(3)$ and $\left(\mathrm{Me}_{3} \mathrm{Si}\right)_{3} \mathrm{Si}-\left(\mathrm{Me}_{3} \mathrm{Si}\right)_{2} \mathrm{Si}-\mathrm{CMe}_{2} \mathrm{SiMe}_{3}$ (4). The chemically but not crystallographically equivalent silicon atoms in 3 and 4 and the two crystallographically independent molecules of 4 all gave rise to separate signals in the solid state ${ }^{29} \mathrm{Si}-\mathrm{MAS}$ NMR spectra. The ${ }^{1} \mathrm{~J}\left({ }^{29} \mathrm{Si},{ }^{29} \mathrm{Si}\right)$ couplings observed in solution facilitated the assignment of ${ }^{29} \mathrm{Si}$ resonances.
\end{abstract}

\section{Zusammenfassung}

Das Reaktionsverhalten von $\left(\mathrm{Me}_{3} \mathrm{Si}\right)_{3} \mathrm{SiLi}(1)$ mit Aceton wurde durch die Strukturanalyse und ${ }^{29} \mathrm{Si}-\mathrm{NMR}$-Untersuchungen von $\mathrm{H}\left(\mathrm{Me}_{3} \mathrm{Si}\right)_{2} \mathrm{Si}-\left(\mathrm{Me}_{3} \mathrm{Si}\right)_{2} \mathrm{Si}-\mathrm{CMe}_{2} \mathrm{SiMe}_{3}(3)$ und $\left(\mathrm{Me}_{3} \mathrm{Si}\right)_{3} \mathrm{Si}-\left(\mathrm{Me}_{3} \mathrm{Si}\right)_{2} \mathrm{Si}-\mathrm{CMe}_{2} \mathrm{SiMe}_{3}$ (4) aufgeklärt. Die zwar chemisch aber nicht kristallographisch äquivalenten Siliciumatome in 3 und 4 und die zwei kristallographisch unabhängigen Moleküle in 4 lassen sich alle im ${ }^{29} \mathrm{Si}-\mathrm{MAS}-\mathrm{CP}$-Spektrum auflösen. Die ${ }^{1} J\left({ }^{29} \mathrm{Si},{ }^{29} \mathrm{Si}\right)$-Kopplungen in Lösung erleichtern dabei das Zuordnen der ${ }^{29} \mathrm{Si}-\mathrm{Reso}-$ nanzen.

\section{Einleitung}

Auf der Suche nach einem gangbaren Weg zur Synthese von Silenen haben wir die Frage aufgeworfen, ob ausgehend von Hydroxyalkylpolysilanen ein der Peterson-Reaktion vergleichbarer Ablauf geeignet ist, Si=C-Systeme aufzubauen (G1. (1)).

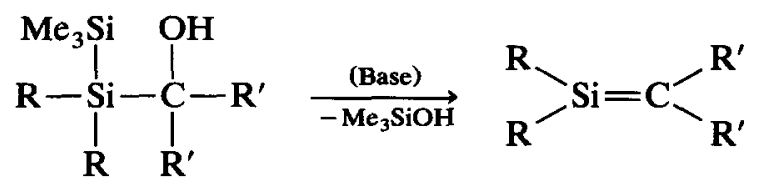

$\mathrm{Zu}$ diesem Zweck hatten wir das Verhalten des Tris(trimethylsilyl)-silyllithiums 1 gegenüber aliphatischen

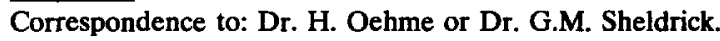

Ketonen, u.a. Aceton, untersucht, deren Primärprodukt der deprotonierten Ausgangsverbindung nach Gl. (1) entspricht ( $\left.\mathrm{R}=\mathrm{SiMe}_{3} ; \mathrm{R}^{\prime}=\mathrm{Me}\right)$ und das zu einem vermutlich instabilen Silen weiterreagieren sollte. Erste Ergebnisse hatten wir mitgeteilt [1,2]; weitergehende Untersuchungen, insbesondere die Röntgenstrukturanalyse zweier aus 1 und Aceton resultierender Reaktionsprodukte, machen jedoch eine teilweise Korrektur der dort gefuihrten Strukturdiskussion erforderlich und führten zu neuen Einsichten in den Ablauf der Reaktion, die Gegenstand dieser Mitteilung sind.

\section{Ergebnisse und Diskussion}

Die Umsetzung von 1 mit Aceton liefert abhängig von der Reaktionsführung unterschiedliche Produkte. Tropft man die THF-Lösung von 1 bei $-40^{\circ} \mathrm{C} z \mathrm{u}$ 


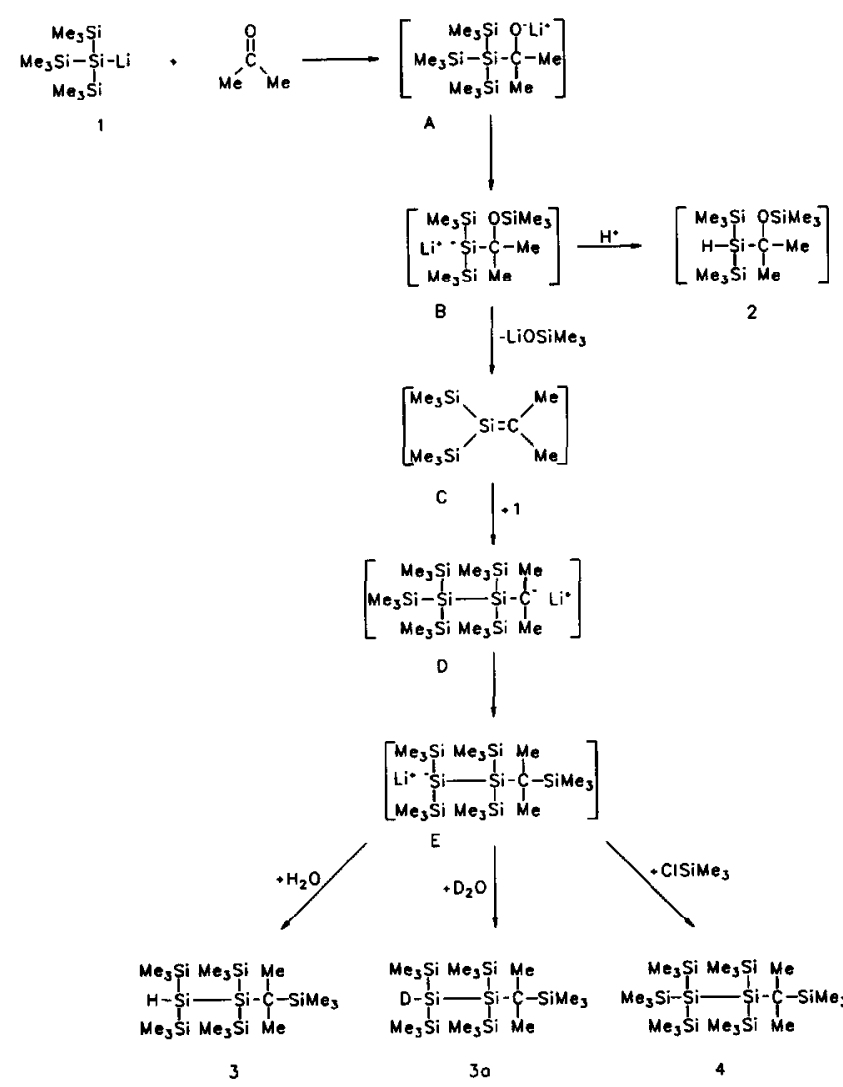

Schema 1.

Aceton, arbeitet man somit also stets bei einem effektiven Überschuß des enolisierbaren Ketons, so erhält man nach hydrolytischer Aufarbeitung 2-[Bis-(trimethylsilyl)-silyl]-2-(trimethylsiloxy)-propan (2). Gibt man unter vergleichbaren Bedingungen Aceton zur Lösung von 1, so resultiert eine farblose kristalline Verbindung, die sich im Ergebnis einer Röntgenkristallstrukturuntersuchung abweichend von der in [2] angegebenen Struktur als 1,1,1,4,4,4-Hexamethyl-2,3-bis(trimethylsilyl)-3-[2-trimethylsilylpropyl(2)]-tetrasilan 3 erwies.
Unsere Vorstellungen über die Bildung von 2 und 3 sind in Schema 1 dargestellt. Danach addiert 1 an die Carbonylfunktion des Acetons zu $\mathbf{A}$, das einer raschen 1,3-Si,O-Trimethylsilylwanderung zu B unterliegt. Umlagerungen dieser Art wurden erstmals von Brook und Crusciel beobachtet [3] und entsprechen einer offenbar generellen Verhaltensweise von $\alpha$-Hydroxyalkylpolysilanen [4]. In Gegenwart überschüssigen Acetons wird B zu 2 protoniert und läßt sich in 42.5-\%iger Ausbeute isolieren. In völlig gleicher Weise reagiert 1 mit weiteren aliphatischen Ketonen zu geminalen Bis-(trimethylsilyl)-silyl-trimethylsiloxy-alkanen [2].

Versuche, das anionische $\mathrm{B}$ durch $\mathrm{D}_{2} \mathrm{O}$ abzufangen bzw. seine Lebensdauer abzuschätzen, führten auch dann, wenn unmittelbar nach erfolgter 1:1-Umsetzung von 1 mit Aceton noch in der Kälte mit $\mathrm{D}_{2} \mathrm{O}$ zersetzt wurde, zu 2, das allenfalls Spuren der deuterierten Verbindung enthielt. Daneben wurde Tris-(trimethylsilyl)silan mit hohen Anteilen von $\left(\mathrm{Me}_{3} \mathrm{Si}\right)_{3} \mathrm{Si}-\mathrm{D}$ nachgewiesen. Das zeigt, daß B durch Aceton sehr viel schneller protoniert wird als 1, das gegenüber Aceton bei tiefen Temperaturen vor allem als Nucleophil fungiert, was angesichts der unterschiedlichen Stabilisierung der negativen Ladungen in den beiden Verbindungen und der somit abgestuften Basizität verständlich erscheint.

In Abwesenheit protonierender Agenzien eliminiert B Lithiumtrimethylsilanolat im Sinne der PetersonReaktion unter gleichzeitiger Bildung des Silens C. Dies gilt z. B. für den Fall, daß Aceton zu 1 getropft wird. Das dabei stets im Überschuß vorhandene Lithiumsilanid 1 addiert an das reaktive $\mathrm{Si}=\mathrm{C}$-System $\mathrm{zu}$ D. Das eliminierte Silanolat wird nach hydrolytischer Aufarbeitung gaschromatographisch als Hexamethyldisiloxan nachgewiesen.

Die Eliminierung von Trimethylsilanolat aus B bzw. vergleichbar strukturierten Derivaten wurde inzwischen auch von anderen Autoren nachgewiesen. So berichten Ishikawa et al. [5] über Umsetzungen $\mathrm{z}$. B. des Acetyl-tris-(trimethylsilyl)silans mit Lithiummethyl

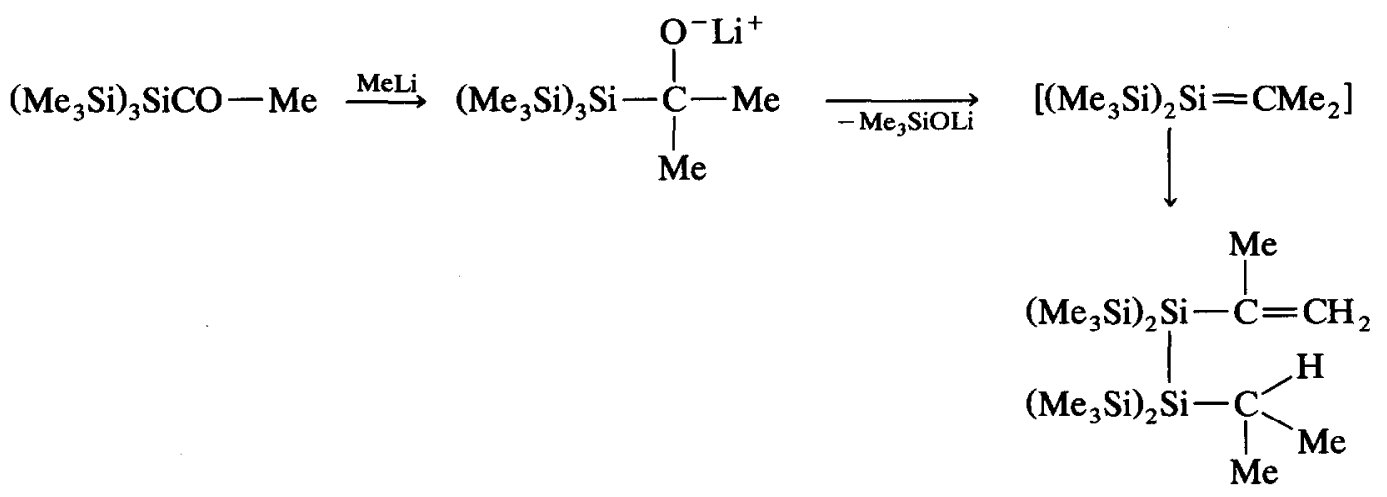


in Diethylether bei $-80^{\circ} \mathrm{C}$, wobei über einen mit $\mathbf{B}$ identischen Zwischenzustand 1-Isopropyl-2-(2-propenyl)-1,1,2,2-tetrakis-(trimethylsilyl)-disilan (5) gebildet wird (Gl. (2)).

Brook et al. diskutieren die Eliminierung von Magnesiumtrimethylsilanolat aus B ähnlichen Strukturen [6], die durch Addition von Grignard-Reagenzien, z.B. von Methylmagnesiumbromid, an das stabile Silen $\left(\mathrm{Me}_{3} \mathrm{Si}\right)_{2} \mathrm{Si}=\mathrm{CAd}(\mathrm{OSiMe})_{3}$ und nachfolgende AntiBrook-Umlagerung gebildet werden. In Abwesenheit stärkerer Nucleophile beobachten die Autoren Readdition des Silanolats an das zunächst entstandene Silen. In Gegenwart überschüssigen Grignard-Reagens konkurriert dies erfolgreich mit dem Silanolat um das Silen, und es erfolgt die erwartete Addition des CNucleophils (Gl. (3)). In einer kürzlich erschienenen Arbeit haben schließlich Apeloig et al. [7] nachgewiesen, daß $\left(\mathrm{Me}_{3} \mathrm{Si}\right)_{3} \mathrm{SiLi} \cdot 3 \mathrm{THF}$ in Hexan oder Benzol mit Adamantanon oder 4-tert-Butylcyclohexanon nach einem der Peterson-Reaktion vergleichbaren Mechanismus zu Silenen $\left(\mathrm{Me}_{3} \mathrm{Si}\right)_{2} \mathrm{Si}=\mathrm{CRSiMe}_{3}(\mathrm{R}=\mathrm{Ad}$; 4 tert- $\mathrm{BuC}_{6} \mathrm{H}_{10}$ ) reagiert, die in einer Kopf-Kopf-Dimerisierung 1,2-Disilacyclobutane liefern.

Das carbanionische Intermediat $\mathbf{D}$ durch Hydrolyse abzufangen, ist bislang nicht gelungen. Es unterliegt offenbar sehr rasch einer Umwandlung in das Silaanion $\mathbf{E}$, das nach Hydrolyse 3, nach Deuterolyse 3a liefert. Die Reaktion von E mit Chlortrimethylsilan führt zu dem Polysilan 4.

Die Umwandlung $D \rightarrow E$ stellt sich summarisch als eine 1,3-Wanderung einer Trimethylsilylgruppierung von einem Silicium- zu einem Kohlenstoffatom dar. Dieser Vorgang erscheint angesichts der besonderen Stabilisierung der negativen Ladung im Silaanion E durch drei benachbarte Si-Atome verständlich. $\mathrm{Ob}$ diesc Isomcrisierung tatsächlich im Sinne einer intramolekularen 1,3-Trimethylsilylverschiebung oder aber
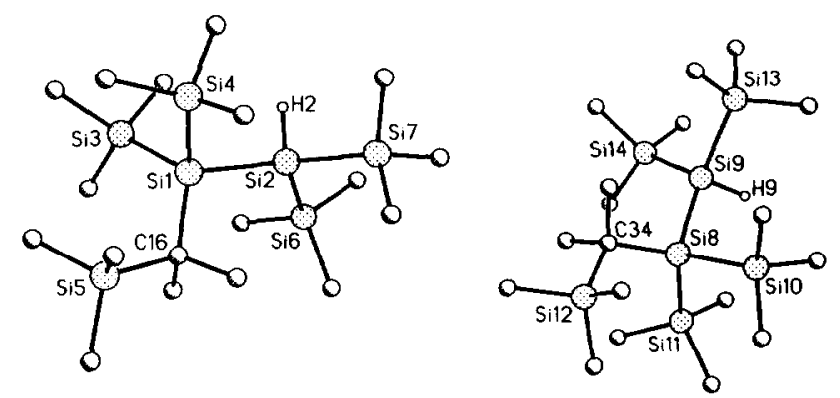

Fig. 1. Kristallstruktur von 3 mit zwei unabhängigen Molekülen in der asymmetrischen Einheit. als bimolekulare Reaktion abläuft, ist auf der Basis des vorliegenden Materials nicht $\mathrm{zu}$ entscheiden.

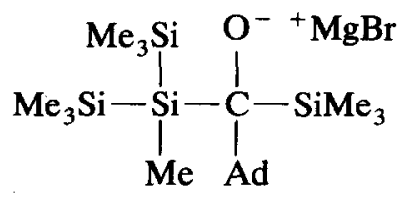

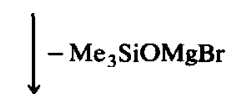<smiles>CCC(C)C(CBr)[SiH](C)O[Mg]</smiles><smiles>C[Si](C)(C)[Si](C)(O[SiH3])C([14CH3])([SiH3])[SiH3]</smiles><smiles>C[SiH2][Si](C)(C)C([AlH2])([SiH3])[SiH2]Br</smiles>

\section{Kristallstrukturen von 3 und 4}

3 kristallisiert aus n-Pentan in der monoklinen Raumgruppe $P 2_{\mathfrak{l}} / c$ mit zwei kristallographisch unabhängigen Molekülen in der asymmetrischen Einheit. Beide Moleküle sind nahezu identisch. Die siliciumgebundenen Wasserstoffatome $\mathrm{H} 2$ und $\mathrm{H} 9$ (Fig. 1) stehen jeweils gestaffelt zu den beiden an Si1 bzw. Si8 gebundenen Trimethylsilylgruppen. Die beiden jeweils an Si2 und Si9 gebundenen Trimethylsilylgruppen stehen gestaffelt zu den $-\mathrm{CMe}_{2} \mathrm{SiMe}_{3}$-Substituenten an $\mathrm{Sil}$ und Si8 und schließen aufgrund des sterischen Drucks den kleinsten (durchschnittlich $107.8^{\circ}$ ) der Si-Si-SiWinkel am Si2 bzw. Si9 ein. Die entsprechenden anderen Winkel sind mit durchschnittlich $119.9^{\circ} \mathrm{ca} .12^{\circ}$ größer. Die $\mathrm{Si}-\mathrm{Si}$-Bindungen sind alle nahezu identisch und betragen im Durchschnitt 237.3 pm. Damit sind sie in guter Übereinstimmung mit den Si-Si-Bindungen in $\left(\mathrm{Me}_{3} \mathrm{Si}\right)_{4} \mathrm{Si}(234.6 \mathrm{pm}$ im Kristall [8] und $236.1 \mathrm{pm}$ in der Gasphase [9]) und $\left(\mathrm{Me}_{3} \mathrm{Si}\right)_{3} \mathrm{Si}$ $\mathrm{Si}\left(\mathrm{SiMe}_{3}\right)_{3}\left(237.3 \mathrm{pm}\right.$ in den $\left(\mathrm{Me}_{3} \mathrm{Si}\right)_{3} \mathrm{Si}$-Gruppen und $239.0 \mathrm{pm}$ in der zentralen Bindung [8]).

Bei 4 dagegen lassen sich in den $\mathrm{Si}-\mathrm{Si}$-Bindungslängen erhebliche Unterschiede feststellen. Die Si-Si-Abstände der zentralen Si1 und Si5-Atome zu den Trimethylsilylgruppen sind zwar nahezu identisch (durchschnittlich $238.5 \mathrm{pm}$ ), jedoch die zentrale Si1-Si5-Bindung ist aufgrund des sterischen Drucks mit $244.3 \mathrm{pm}$ ca. $6 \mathrm{pm}$ länger (Fig. 2, Tab. 2). Sie ist sogar $5 \mathrm{pm}$ länger als die zentrale Bindung in $\left(\mathrm{Me}_{3} \mathrm{Si}\right)_{3} \mathrm{Si}-$ $\mathrm{Si}\left(\mathrm{SiMe}_{3}\right)_{3}[8]$. Darüberhinaus ist der durchschnittliche 


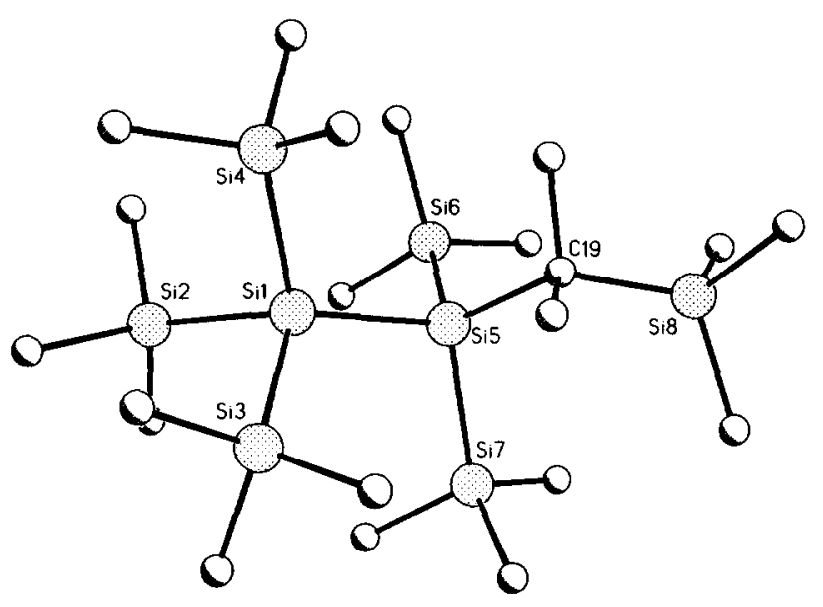

Fig. 2. Kristallstruktur von 4.

$\mathrm{Me}_{3} \mathrm{Si}-\mathrm{Si}-\mathrm{SiMe}_{3}$-Winkel am Si1 mit $102.4^{\circ}$ bemerkenswert klein und nahezu identisch mit dem entsprechenden Winkel in $\left(\mathrm{Me}_{3} \mathrm{Si}\right)_{3} \mathrm{SiLi}(\mathrm{THF})_{3}$ [8]. Der durchschnittliche $\mathrm{Me}_{3} \mathrm{Si}-\mathrm{Si}-\mathrm{Si}\left(\mathrm{SiMe}_{3}\right)_{2} \mathrm{CMe}_{2}$-Winkel ist mit $115.9^{\circ}$ erheblich größer. Der sterische Anspruch der $\mathrm{Si}\left(\mathrm{SiMe}_{3}\right)_{2} \mathrm{CMe}_{2} \mathrm{SiMe}_{3}$-Gruppe ist offensichtlich größer als der der $\mathrm{Si}\left(\mathrm{SiMe}_{3}\right)_{3}$-Gruppe, da hier der $\mathrm{Me}_{3} \mathrm{Si}-\mathrm{Si}-\mathrm{SiMe}_{3}$-Winkel $104.8^{\circ}$ beträgt. In den Verbindungen $\left[\mathrm{Li}(\mathrm{THF})_{4}\right]\left[\mathrm{Cl}_{3} \mathrm{AlSi}\left(\mathrm{SiMe}_{3}\right)_{3}\right]$ und $[\mathrm{Li}-$ (THF) $\left.\left.{ }_{A}\right]\left[\mathrm{Cu}_{5} \mathrm{Cl}_{4}\left(\mathrm{Si}_{(\mathrm{SiMe}}\right)_{3}\right)_{2}\right]$ ist der entsprechende Winkel $110^{\circ}[10]$. Im Gegensatz zu den beiden letztgenannten Verbindungen und $\mathrm{zu}\left(\mathrm{Me}_{3} \mathrm{Si}\right)_{3} \mathrm{SiLi}(\mathrm{THF})_{3}$ muß man in 4 wohl ausschließlich sterische Gründe für die kleineren $\mathrm{Si}-\mathrm{Si}-\mathrm{Si}$-Winkel verantwortlich machen. Kristallographische Daten sind in den Tab. 1-4 angegeben.

\section{4. ${ }^{29}$ Si-NMR-spektroskopische Untersuchungen in Lösung und im Festkörper}

Das ${ }^{29} \mathrm{Si}-\mathrm{NMR}-\mathrm{Spektrum}$ von 3 aus Lösung (Fig. 3(b), Tab. 5) zeigt die aufgrund der Röntgenstrukturanalyse erwarteten fünf Signale. Das wasserstoffgebundene Siliciumatom tritt bei $-115.2 \mathrm{ppm}$ in Resonanz, was durch die INEPT-Messung zweifelsfrei belegt werden kann. Das zentrale kohlenstoffgebundene Siliciumatom tritt bei $-45.6 \mathrm{ppm}$ und das der peripheren kohlenstoffgebundenen $\mathrm{Me}_{3} \mathrm{Si}$-Gruppe bei $8.4 \mathrm{ppm}$ in Resonanz. Die übrigen geminalen Trimethylsilylgruppen zeigen jeweils ein Signal bei -13.5 und -10.1

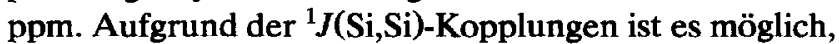
diese Signale zuzuordnen: das Signal bei -13.5 ppm entspricht den $\mathrm{Si}\left(\mathrm{SiMe}_{3}\right)_{2} \mathrm{CMe}_{2}$-Gruppen, das Signal bei $-10.1 \mathrm{ppm}$ den $\mathrm{HSi}\left(\mathrm{SiMe}_{3}\right)_{2}$-Gruppen (Tab. 5).

Das ${ }^{29}$ Si-MAS-NMR-Spektrum von 3 (Fig. 3(a)) zeigt ein Phänomen, das nicht zu verstehen ist, wenn man das Ergebnis der Strukturanalyse nicht kennt: aufgrund der zwei im Kristall vorhandenen unabhängigen Moleküle kommt es zu einer Signalverdopplung. Die Signale, die nur einem Siliciumatom entsprechen, sind

TABELLE 1. Atomkoordinaten $\left(\times 10^{4}\right)$ und äquivalente isotrope Auslenkungsparameter $\left(\mathrm{pm}^{2} \times 10^{-1}\right)$ von 3 . $U_{\text {eq }}$ wird berechnet als ein Drittel der Spur des orthogonalen $U_{i}$-Tensors

\begin{tabular}{|c|c|c|c|c|}
\hline Atom & $x$ & $y$ & $z$ & $U_{\text {eq }}$ \\
\hline$\overline{\text { Si(1) }}$ & $4655.6(4)$ & $5643.0(4)$ & $7150.1(4)$ & $23.7(4)$ \\
\hline $\mathrm{Si}(2)$ & $4148.6(4)$ & $6744.2(4)$ & $6611.5(5)$ & $26.5(4)$ \\
\hline $\mathrm{H}(2)$ & $4721(16)$ & $7167(14)$ & $7141(16)$ & $48(8)$ \\
\hline $\mathrm{Si}(3)$ & $5108.5(5)$ & $5750.7(4)$ & $8571.7(5)$ & $37.8(6)$ \\
\hline $\mathrm{Si}(4)$ & $5829.7(5)$ & $5561.8(4)$ & $7022.3(5)$ & $32.6(4)$ \\
\hline $\mathrm{Si}(5)$ & $4156.3(5)$ & $4046.2(4)$ & $6774.9(5)$ & $37.5(6)$ \\
\hline $\operatorname{Si}(6)$ & $3001.5(5)$ & $7127.1(4)$ & $6665.3(6)$ & $40.1(5)$ \\
\hline $\mathrm{Si}(7)$ & $4100.7(5)$ & $7123.0(4)$ & $5382.0(5)$ & $36.7(5)$ \\
\hline$C(1)$ & $6048(2)$ & $5277(2)$ & $9198(2)$ & $52(2)$ \\
\hline$C(2)$ & $4395(2)$ & $5478(2)$ & $8947(2)$ & $59(3)$ \\
\hline$C(3)$ & $5364(2)$ & $6668(2)$ & $8857(2)$ & $56(3)$ \\
\hline C(4) & $6398(2)$ & $6366(2)$ & $7456(2)$ & $51(2)$ \\
\hline$C(5)$ & $6498(2)$ & $4838(2)$ & $7584(2)$ & $66(2)$ \\
\hline$C(6)$ & $5659(2)$ & $5479(2)$ & $5953(2)$ & $47(2)$ \\
\hline$C(7)$ & $3257(2)$ & $3497(2)$ & $6403(2)$ & $69(3)$ \\
\hline$C(8)$ & $4757(2)$ & $3855(2)$ & $7868(2)$ & $56(3)$ \\
\hline$c(9)$ & $4707(2)$ & $3775(2)$ & $6220(2)$ & $64(3)$ \\
\hline$C(10)$ & $2084(2)$ & $6821(2)$ & $5794(2)$ & $76(2)$ \\
\hline $\mathrm{C}(11)$ & $2982(2)$ & $6885(2)$ & $7623(2)$ & $63(2)$ \\
\hline $\mathrm{C}(12)$ & $2992(2)$ & $8079(2)$ & $6654(3)$ & $70(3)$ \\
\hline$C(13)$ & $3540(2)$ & $7940(2)$ & $5066(2)$ & $67(3)$ \\
\hline C(14) & $5116(2)$ & $7329(2)$ & $5531(2)$ & $54(2)$ \\
\hline$C(15)$ & $3618(2)$ & $6523(2)$ & $4512(2)$ & $57(3)$ \\
\hline$C(16)$ & $3844(2)$ & $4973.2(14)$ & $6590(2)$ & $31(2)$ \\
\hline $\mathrm{C}(17)$ & $3496(2)$ & $5111(2)$ & $5678(2)$ & $47(2)$ \\
\hline$C(18)$ & $3181(2)$ & $5077(2)$ & $6835(2)$ & $47(2)$ \\
\hline $\mathrm{Si}(8)$ & $316.5(4)$ & $10606.4(4)$ & $2843.0(4)$ & $24.7(4)$ \\
\hline Si(9) & $917.7(4)$ & $11686.2(4)$ & $3359.3(4)$ & $25.9(4)$ \\
\hline $\mathrm{H}(9)$ & $340(15)$ & $12139(13)$ & $2831(15)$ & $38(8)$ \\
\hline Si(10) & $222.8(5)$ & $10584.1(5)$ & $1534.8(5)$ & $34.8(5)$ \\
\hline Si(11) & $-1007.2(5)$ & $10740.2(4)$ & $2641.2(5)$ & $33.9(4)$ \\
\hline $\mathrm{Si}(12)$ & $664.4(5)$ & $8989.7(4)$ & $3203.5(6)$ & $44.4(5)$ \\
\hline Si(13) & $2065.7(5)$ & $12049.6(4)$ & $3271.2(5)$ & $33.7(5)$ \\
\hline Si(14) & $1080.2(5)$ & $12027.0(4)$ & $4639.8(5)$ & $35.7(5)$ \\
\hline C(19) & $-454(2)$ & $9916(2)$ & $833(2)$ & $62(2)$ \\
\hline$C(20)$ & $-195(2)$ & $11425(2)$ & $1060(2)$ & $52(2)$ \\
\hline$C(21)$ & $1207(2)$ & $10469(2)$ & $1552(2)$ & $47(2)$ \\
\hline $\mathrm{C}(22)$ & $-1193(2)$ & $10411(2)$ & $3481(2)$ & $51(2)$ \\
\hline $\mathrm{C}(23)$ & $-1763(2)$ & $10330(2)$ & $1689(2)$ & $52(2)$ \\
\hline$C(24)$ & $-1268(2)$ & $11670(2)$ & $2514(2)$ & $53(2)$ \\
\hline$C(25)$ & $-411(2)$ & $8831(2)$ & $2746(2)$ & $60(2)$ \\
\hline$C(26)$ & $1016(2)$ & $8763(2)$ & $2460(3)$ & $74(3)$ \\
\hline$C(27)$ & $1155(2)$ & $8390(2)$ & $4069(3)$ & $77(3)$ \\
\hline$C(28)$ & $2478(2)$ & $12836(2)$ & $3896(2)$ & $56(2)$ \\
\hline$C(29)$ & $2891(2)$ & $11420(2)$ & $3620(2)$ & $52(2)$ \\
\hline$C(30)$ & $1819(2)$ & $12312(2)$ & $2215(2)$ & $46(2)$ \\
\hline$C(31)$ & $307(2)$ & $11722(2)$ & $4919(2)$ & $60(3)$ \\
\hline$C(32)$ & $2060(2)$ & $11726(2)$ & $5436(2)$ & $53(2)$ \\
\hline$C(33)$ & $1030(2)$ & $12977(2)$ & $4655(2)$ & $54(2)$ \\
\hline$C(34)$ & $932(2)$ & $9895.7(14)$ & $3577(2)$ & $32(2)$ \\
\hline$C(35)$ & $1808(2)$ & $10014(2)$ & $3787(2)$ & $47(2)$ \\
\hline$C(36)$ & $863(2)$ & $9966(2)$ & $4365(2)$ & $48(2)$ \\
\hline
\end{tabular}


TABELLE 2. Atomkoordinaten $\left(\times 10^{4}\right)$ und äquivalente isotrope Auslenkungsparameter $\left(\mathrm{pm}^{2} \times 10^{-1}\right)$ von $4 . U_{\text {eq }}$ wird berechnet als ein Drittel der Spur des orthogonalen $U_{i j}$-Tensors

\begin{tabular}{lrrrl}
\hline Atom & \multicolumn{1}{l}{$\boldsymbol{x}$} & \multicolumn{1}{l}{$y$} & $U_{\text {eq }}$ \\
\hline $\mathrm{Si}(1)$ & $7193.0(5)$ & $8721.3(5)$ & $8501.5(2)$ & $16.3(3)$ \\
$\mathrm{Si}(2)$ & $7158.7(6)$ & $8761.6(5)$ & $7574.7(2)$ & $21.3(3)$ \\
$\mathrm{Si}(3)$ & $7879.3(6)$ & $6865.3(5)$ & $8667.4(2)$ & $21.7(3)$ \\
$\mathrm{Si}(4)$ & $5124.5(6)$ & $8644.6(6)$ & $8696.5(3)$ & $22.5(3)$ \\
$\mathrm{Si}(5)$ & $8292.7(5)$ & $10216.5(5)$ & $8933.1(2)$ & $17.6(3)$ \\
$\mathrm{Si}(6)$ & $7925.7(6)$ & $11934.1(5)$ & $8488.8(3)$ & $22.7(3)$ \\
$\mathrm{Si}(7)$ & $10357.0(6)$ & $9901.6(6)$ & $8837.4(3)$ & $25.0(3)$ \\
Si(8) & $8672.9(7)$ & $11045.2(6)$ & $10180.4(3)$ & $31.8(4)$ \\
$\mathrm{C}(1)$ & $8598(2)$ & $9012(2)$ & $7229.7(9)$ & $32.5(14)$ \\
$\mathrm{C}(2)$ & $6670(3)$ & $7381(2)$ & $7299.4(9)$ & $31.2(14)$ \\
$\mathrm{C}(3)$ & $6048(3)$ & $9815(2)$ & $7343.3(10)$ & $34.4(15)$ \\
$\mathrm{C}(4)$ & $9218(3)$ & $6556(2)$ & $8265.4(11)$ & $34.5(14)$ \\
$\mathrm{C}(5)$ & $8272(3)$ & $6490(2)$ & $9353.6(10)$ & $36(2)$ \\
$\mathrm{C}(6)$ & $6712(3)$ & $5826(2)$ & $8482.4(11)$ & $32.8(14)$ \\
$\mathrm{C}(7)$ & $4289(3)$ & $7728(2)$ & $8232.0(12)$ & $37.4(13)$ \\
$\mathrm{C}(8)$ & $4298(2)$ & $9998(2)$ & $8652.6(12)$ & $33.8(11)$ \\
$\mathrm{C}(9)$ & $4847(3)$ & $8047(3)$ & $9359.1(11)$ & $37.7(14)$ \\
$\mathrm{C}(10)$ & $8517(3)$ & $11853(2)$ & $7811.5(10)$ & $39(2)$ \\
$\mathrm{C}(11)$ & $8678(3)$ & $13192(2)$ & $8773.8(12)$ & $40(2)$ \\
$\mathrm{C}(12)$ & $6326(2)$ & $12316(2)$ & $8433.6(11)$ & $34.7(14)$ \\
$\mathrm{C}(13)$ & $10927(3)$ & $8715(2)$ & $9244.0(11)$ & $37.9(14)$ \\
$\mathrm{C}(14)$ & $10808(3)$ & $9557(3)$ & $8156.5(10)$ & $36.4(13)$ \\
$\mathrm{C}(15)$ & $11173(2)$ & $11228(2)$ & $8994.3(13)$ & $39.9(12)$ \\
$\mathrm{C}(16)$ & $8793(3)$ & $12582(2)$ & $10078.1(12)$ & $43(2)$ \\
$\mathrm{C}(17)$ & $10189(3)$ & $10481(3)$ & $10310.6(13)$ & $51(2)$ \\
$\mathrm{C}(18)$ & $7854(4)$ & $10885(3)$ & $10811.2(11)$ & $58(3)$ \\
$\mathrm{C}(19)$ & $7804(2)$ & $10264(2)$ & $9659.4(8)$ & $23.1(11)$ \\
$\mathrm{C}(20)$ & $6542(2)$ & $10761(2)$ & $9669.7(10)$ & $30.3(12)$ \\
$\mathrm{C}(21)$ & $7741(3)$ & $9040(2)$ & $9853.1(9)$ & $32(2)$ \\
\hline & & & & \\
\hline
\end{tabular}

im Festkörperspektrum einfach gespalten. Im Bereich -9.5 bis $-14.5 \mathrm{ppm}$ zeigt sich, daß nicht nur die geminalen $\mathrm{Me}_{3} \mathrm{Si}$-Gruppen nicht mehr äquivalent sind, sondern daß darüberhinaus acht Signale für acht magnetisch unterschiedliche Trimethylsilylgruppen aufzulösen sind. Hier liegt der unseres Wissens erste Fall vor, wo im ${ }^{29}$ Si-MAS-NMR-Spektrum zwei kristallographisch unabhängige Moleküle aufgelöst werden konnten. Nur aufgrund des ${ }^{29}$ Si-MAS-NMR-Spektrums wäre es jedoch vermutlich nicht zu einem Strukturvorschlag, so wie er in der Strukturanalyse von 3 gefunden wurde, gekommen. Uns scheint es viel einfacher, ein ${ }^{29}$ Si-MAS-NMR-Spektrum nach zweifelsfrei vorliegender Struktur zu interpretieren, als eine zweifelsfreie Struktur aus dem Spektrum abzuleiten.

Das ${ }^{29}$ Si-NMR-Spektrum von 4 aus Lösung (Fig. 4(b), Tab. 5) zeigt ebenfalls die nach der Strukturanalyse erwarteten fünf Signale. Das an drei $\mathrm{Me}_{3} \mathrm{Si}$-Gruppen gebundene zentrale Siliciumatom tritt bei -126.5 ppm in Resonanz. Diese Verschiebung zu hohem Feld ist typisch [8-12]. Das an zwei $\mathrm{Me}_{3} \mathrm{Si}-$ Gruppen ge-
TABELLE 3. Ausgewählte Bindungslängen (pm) und -winkel $\left(^{\circ}\right)$ von 3

\begin{tabular}{llll}
\hline Si(1)-C(16) & $192.8(3)$ & Si(1)-Si(4) & $235.95(12)$ \\
Si(1)-Si(2) & $238.90(12)$ & Si(1)-Si(3) & $238.90(12)$ \\
Si(2)-Si(6) & $235.46(12)$ & Si(2)-Si(7) & $236.97(12)$ \\
Si(2)-H(2) & $136.4(27)$ & Si(5)-C(16) & $189.3(3)$ \\
C(16)-C(17) & $153.8(4)$ & C(16)-C(18) & $154.0(4)$ \\
Si(8)-C(34) & $192.7(3)$ & Si(8)-Si(10) & $236.14(12)$ \\
Si(8)-Si(11) & $238.56(12)$ & Si(8)-Si(9) & $238.82(12)$ \\
Si(9)-Si(14) & $235.73(12)$ & Si(9)-Si(13) & $237.50(12)$ \\
Si(9)-H(9) & $140.4(25)$ & Si(12)-C(34) & $189.0(3)$ \\
C(34)-C(36) & $153.9(4)$ & C(34)-C(35) & $154.7(4)$ \\
Si(4)-Si(1)-Si(2) & $103.73(4)$ & C(16)-Si(1)-Si(3) & $116.89(9)$ \\
Si(4)-Si(1)-Si(3) & $103.96(4)$ & Si(2)-Si(1)-Si(3) & $104.86(4)$ \\
Si(6)-Si(2)-Si(7) & $108.45(4)$ & Si(6)-Si(2)-Si(1) & $118.29(4)$ \\
Si(7)-Si(2)-Si(1) & $121.20(4)$ & Si(6)-Si(2)-H(2) & $102.9(11)$ \\
Si(7)-Si(2)-H(2) & $100.0(11)$ & Si(1)-Si(2)-H(2) & $102.1(11)$ \\
C(17)-C(16)-C(18) & $108.0(2)$ & C(17)-C(16)-Si(5) & $108.0(2)$ \\
C(18)-C(16)-Si(5) & $107.7(2)$ & $C(17)-C(16)-S i(1)$ & $108.1(2)$ \\
C(18)-C(16)-Si(1) & $108.2(2)$ & Si(5)-C(16)-Si(1) & $116.63(15)$ \\
Si(10)-Si(8)-Si(11) & $105.39(4)$ & $C(34)-S i(8)-S i(9)$ & $109.39(9)$ \\
Si(10)-Si(8)-Si(9) & $103.14(4)$ & Si(11)-Si(8)-Si(9) & $104.03(4)$ \\
Si(14)-Si(9)-Si(13) & $106.71(4)$ & Si(14)-Si(9)-Si(8) & $118.34(4)$ \\
Si(13)-Si(9)-Si(8) & $121.61(4)$ & Si(14)-Si(9)-H(9) & $103.2(10)$ \\
Si(13)-Si(9)-H(9) & $102.2(10)$ & Si(8)-Si(9)-H(9) & $101.6(10)$ \\
C(36)-C(34)-C(35) & $107.4(2)$ & C(36)-O(34)-Si(12) & $108.0(2)$ \\
C(35)-C(34)-Si(12) & $108.1(2)$ & C(36)-C(34)-Si(8) & $108.8(2)$ \\
C(35)-C(34)-Si(8) & $107.7(2)$ & Si(12)-C(34)-Si(8) & $116.41(15)$ \\
\hline & & &
\end{tabular}

bundene Siliciumatom tritt bei $-33.4 \mathrm{ppm}$ und das kohlenstoffgebundene Siliciumatom bei $9.0 \mathrm{ppm}$ in Resonanz. Die drei $\mathrm{Me}_{3} \mathrm{Si}$-Gruppen zeigen ein Signal bei $-8.6 \mathrm{ppm}$, die zwei am Si5 gebundenen (Fig. 2) bei -14.2 ppm. Im ${ }^{29}$ Si-MAS-NMR-Spektrum (Fig. 4(a)) spalten nur die Signale auf, die zwei bzw. drei Trimethylsilylgruppen entsprechen. Das Signal für die beiden geminalen $\mathrm{Me}_{3} \mathrm{Si}$-Gruppen zeigt zwei, das der drei

TABELLE 4. Ausgewählte Bindungslängen (pm) und -winkel $\left(^{\circ}\right)$ von 4

\begin{tabular}{llll}
\hline Si(1)-Si(2) & $238.72(10)$ & Si(1)-Si(3) & $238.77(11)$ \\
Si(1)-Si(4) & $239.07(11)$ & Si(1)-Si(5) & $244.31(11)$ \\
Si(5)-C(19) & $195.1(2)$ & Si(5)-Si(7) & $237.38(11)$ \\
Si(5)-Si(6) & $238.60(11)$ & Si(8)-C(18) & $187.9(3)$ \\
Si(8)-C(19) & $190.6(2)$ & C(19)-C(20) & $154.4(3)$ \\
C(19)-C(21) & $154.7(3)$ & & \\
Si(2)-Si(1)-Si(3) & $101.70(3)$ & Si(2)-Si(1)-Si(4) & $101.24(4)$ \\
Si(3)-Si(1)-Si(4) & $104.11(4)$ & Si(2)-Si(1)-Si(5) & $116.63(3)$ \\
Si(3)-Si(1)-Si(5) & $115.65(4)$ & Si(4)-Si(1)-Si(5) & $115.41(3)$ \\
C(19)-Si(5)-Si(7) & $112.45(8)$ & C(19)-Si(5)-Si(6) & $112.68(8)$ \\
Si(7)-Si(5)-Si(6) & $104.90(4)$ & C(19)-Si(5)-Si(1) & $108.28(8)$ \\
Si(7)-Si(5)-Si(1) & $109.63(3)$ & Si(6)-Si(5)-Si(1) & $108.82(3)$ \\
O(20)-C(19)-C(21) & $108.4(2)$ & C(20)-C(19)-Si(8) & $105.9(2)$ \\
C(21)-C(19)-Si(8) & $105.0(2)$ & $C(20)-C(19)-S i(5)$ & $106.8(2)$ \\
C(21)-C(19)-Si(5) & $107.14(15)$ & Si(8)-C(19)-Si(5) & $122.92(13)$ \\
\hline
\end{tabular}



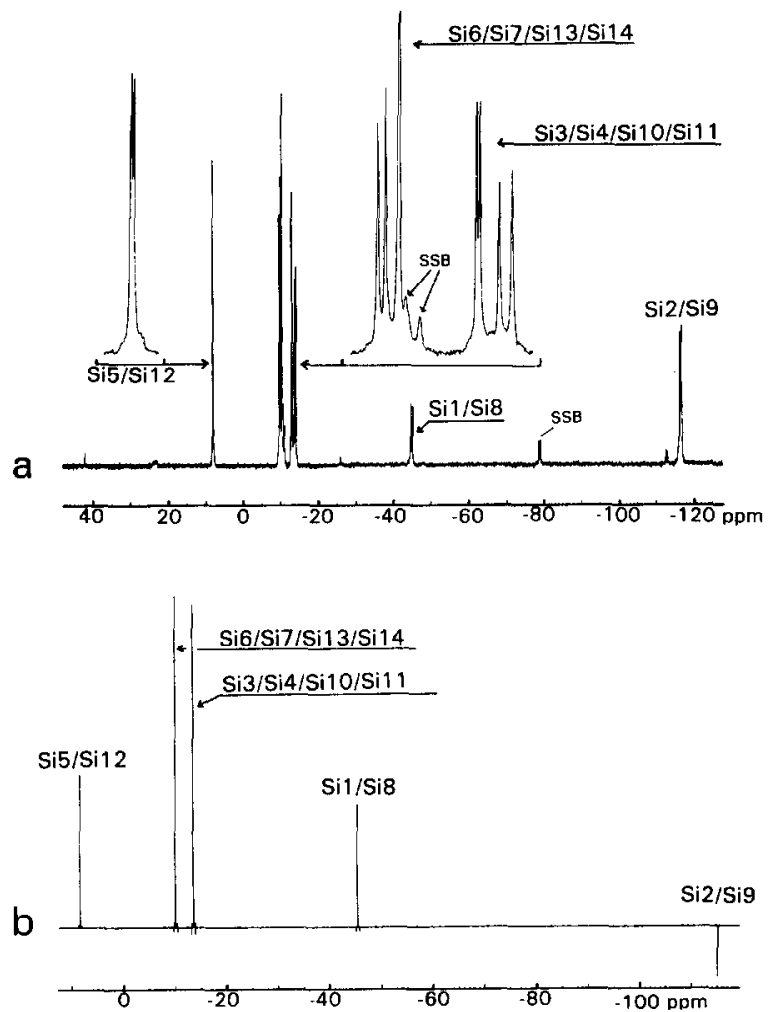

Fig. 3. (a) ${ }^{29} \mathrm{Si}-\mathrm{CP}-\mathrm{MAS}-\mathrm{NMR}-\mathrm{Spektrum}$ von 3 bei einer Rotation von $2700 \mathrm{~Hz}$; (b) ${ }^{29} \mathrm{Si}$-INEPT-NMR-Spektrum von 3 in Pentan $/ \mathrm{C}_{6} \mathrm{D}_{6}$.

$\mathrm{Me}_{3} \mathrm{Si}-\mathrm{Gruppen}$ am Si1 drei unterschiedliche chemische Verschiebungen.

\section{Kristallographische Daten der Verbindung 3}

Ein farbloser Kristall der Größe $0.8 \times 0.7 \times 0.6 \mathrm{~mm}$ wurde bei $-85^{\circ} \mathrm{C}$ auf einem Stoe-Siemens-Vierkreisdiffraktometer mit monochromatisierter Mo K $\alpha$-Strahlung ( $\lambda 71.073 \mathrm{pm}$ ) vermessen. 3 kristallisiert in der Raumgruppe $P 2_{1} / c$ mit den Zellkonstanten $a$

TABELLE 5. ${ }^{29} \mathrm{Si}-\mathrm{NMR}$-Verschiebungen (ppm) und Kopplungen $(\mathrm{Hz})$ von 3 und 4 aus Lösung

\begin{tabular}{|c|c|c|c|}
\hline & 3 & 4 & \\
\hline $\begin{array}{l}\delta(\mathrm{SiH}) \\
\delta\left(\mathrm{HSi}\left(\mathrm{SiMe}_{3}\right)_{2}\right) \\
{ }^{1} J(\mathrm{Si}, \mathrm{Si}) \\
{ }^{1} J(\mathrm{Si}, \mathrm{C})\end{array}$ & $\begin{array}{r}-115.2 \\
-10.1 \\
57.6 \\
44.9\end{array}$ & $\begin{array}{r}-126.5 \\
-8.6 \\
47.6 \\
44.3\end{array}$ & $\begin{array}{l}\delta\left(\mathrm{Si}_{\left.\left(\mathrm{SiMe}_{3}\right)_{3}\right)}\right. \\
\left.\delta\left(\mathrm{Si}(\mathrm{SiMe})_{3}\right)_{3}\right) \\
{ }^{1} J(\mathrm{Si}, \mathrm{Si}) \\
{ }^{1} J(\mathrm{Si}, \mathrm{C})\end{array}$ \\
\hline $\begin{array}{l}\delta\left(\mathrm{SiCMe}_{2} \mathrm{SiMe}_{3}\right) \\
\delta\left(\mathrm{Si}\left(\mathrm{Si} \mathrm{Me}_{3}\right)_{2} \mathrm{CMe}_{2}\right) \\
{ }^{1} J(\mathrm{Si}, \mathrm{Si}) \\
{ }^{1} J(\mathrm{Si}, \mathrm{C})\end{array}$ & $\begin{array}{r}-45.4 \\
-13.5 \\
57.2 \\
44.4\end{array}$ & $\begin{array}{r}-33.4 \\
-14.2 \\
56.7 \\
43.9\end{array}$ & $\begin{array}{l}\delta\left(\mathrm{SiCMe}_{2} \mathrm{SiMe}_{3}\right) \\
\left.\delta\left(\mathrm{Si}(\mathrm{Si} \mathrm{Me})_{3}\right)_{2} \mathrm{CMe}_{2}\right) \\
{ }_{J} J(\mathrm{Si}, \mathrm{Si}) \\
{ }_{J} J(\mathrm{Si}, \mathrm{C})\end{array}$ \\
\hline $\begin{array}{l}\delta\left(\mathrm{Me}_{3} \mathrm{SiCMe}_{2}\right) \\
{ }^{1} J\left(\mathrm{Si}, \mathrm{Me}_{3}\right) \\
{ }^{1} J\left(\mathrm{HSi}_{2} \mathrm{SiSiSi}_{2} \mathrm{CMc}_{2}\right)\end{array}$ & $\begin{array}{r}8.4 \\
50.5 \\
38.1\end{array}$ & $\begin{array}{r}9.0 \\
50.6 \\
30.6\end{array}$ & $\begin{array}{l}\delta\left(\mathrm{Me}_{3} \mathrm{SiCMe}_{2}\right) \\
{ }^{1} J\left(\mathrm{Si}_{\mathrm{Me}}\right) \\
{ }^{1} \mathrm{~J}\left(\mathrm{Si}_{3} \mathrm{SiSiSi}_{2} \mathrm{CMe}_{2}\right)\end{array}$ \\
\hline
\end{tabular}

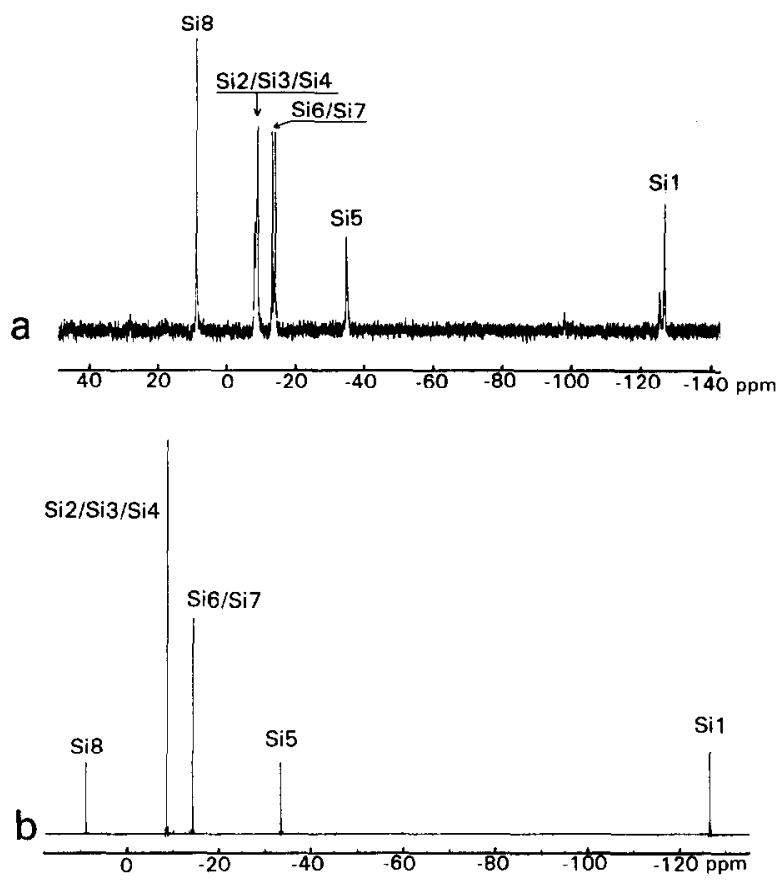

Fig. 4. (a) ${ }^{29}$ Si-CP-MAS-NMR-Spektrum von 4 bei einer Rotation von $5000 \mathrm{~Hz}$; (b) ${ }^{29}$ Si-INEPT-NMR-Spektrum von 4 in Pentan $/ C_{6} D_{6}$.

1903.9(2), $b$ 1959.8(3), c 1863.2(2) pm, $\beta$ 117.32(1),$V$ $6.176 \mathrm{~nm}^{3}, Z=8, \rho_{\text {ber. }} 1.001 \mathrm{Mg} \mathrm{m}^{-3}, \mu 0.312 \mathrm{~mm}^{-1}$ und $F(000)=2064$. Es wurden 8171 Reflexe in einem $2 \Theta$-Bereich von $8-45^{\circ}$ gemessen, davon waren 8079 unabhängige und 8064 wurden für alle Berechnungen verwendet. Die Anzahl der verfeinerten Parameter beträgt 529. Die $R$-Werte sind $R 1=\sum\left|F_{\mathrm{o}}-F_{\mathrm{c}}\right| / \sum F_{\mathrm{o}}=$ $0.041(F>4 \sigma F)$ und $w R 2=\left[\sum w\left(F_{\mathrm{o}}^{2}-F_{\mathrm{c}}^{2}\right)^{2} / \sum w F_{\mathrm{o}}^{4}\right]^{1 / 2}$ $=0.150$ (sämtliche Daten) für $w^{-1}=\sigma^{2}\left(F_{\mathrm{o}}^{2}\right)+(g 1$. $P)^{2}+g 2 \cdot P$ mit $P=\left(F_{\mathrm{o}}^{2}+2 F_{\mathrm{c}}^{2}\right) / 3$ und $g 1=0.031, g 2$ $=4.43$. Maximale- und minimale Restelektronendichte betragen 304 und $-185 \mathrm{e} \mathrm{nm}^{-3}$.

\section{Kristallographische Daten der Verbindung 4}

Ein farbloser Kristall der Größe $0.7 \times 0.7 \times 0.5 \mathrm{~mm}$ wurde auf einem Stoe-Siemens-Vierkreisdiffraktometer mit monochromatisierter Mo $\mathrm{K} \alpha$-Strahlung ( $\lambda$ $71.073 \mathrm{pm}$ ) bei $-120^{\circ} \mathrm{C}$ vermessen. 4 kristallisiert in der Raumgruppe $P 2{ }_{1} 2_{1} 2_{1}$ mit den Zellkonstanten $a$ 1129.1(1), b 1194.9(2), c 2574.9(5) pm, $V 3.474 \mathrm{~nm}^{3}$, $Z=4, \rho_{\text {ber. }} 1.028 \mathrm{Mg} \mathrm{m}^{-3}, \mu 0.318 \mathrm{~mm}^{-1}$ und $F(000)$ $=1192$. Es wurden 5225 Reflexe in einem $2 \Theta$-Bereich von $8-55^{\circ}$ gemessen, davon waren 4814 unabhängige und 4810 wurden für alle Berechnungen verwendet. Die Anzahl der verfeinerten Parameter betrug 303. Die $R$-Werte sind $R 1=0.032(F>4 \sigma F)$ und $w R 2=$ 0.108 (alle Daten) für $g 1=0.044$ und $g 2=0.93$. Die maximale- und minimale Restelektronendichte betra- 
gen 373 bzw. $-241 \mathrm{e} \mathrm{nm}^{-3}$. Die absolute Struktur wurde durch Verfeinerung des Flack Parameters [13] auf $0.03(12)$ bestimmt. Eine semiempirische Absorptionskorrektur wurde durchgeführt.

Die Strukturen wurden mit Hilfe Direkter Methoden (sHelXs-90) [14] gelöst. Sie wurden nach dem Kleinste-Quadrate-Verfahren an $F^{2}$ mit allen Daten verfeinert (sHELXL-92) [15]. Alle Nichtwasserstoffatome wurden anisotrop verfeinert. Die Wasserstoffatome wurden geometrisch ideal positioniert und ihre Lagen nach dem Reitermodell in die Verfeinerung einbezogen. Die Auslenkungsparameter und Bindungslängen äquivalenter Wasserstoffatome wurden gemeinsam frei verfeinert. Die an $\mathrm{Si} 2$ und $\mathrm{Si} 9$ gebundenen Wasserstoffatome wurden völlig frei verfeinert. Weitere Einzelheiten zur Röntgenstrukturanalyse können beim Fachinformationszentrum Karlsruhe, Gesellschaft für wissenschaftlich-technische Information mbH, W-7514 Eggenstein-Leopoldshafen 2, unter Angabe der Hinterlegungsnummer CSD-56688, der Autoren und des Zeitschriftenzitats angefordert werden.

\section{Dank}

Für die Förderung dieser Arbeit danken wir der Deutschen Forschungsgemeinschaft und dem Fonds der Chemischen Industrie. Herrn Wolfgang Zolke danken wir für die Aufnahme der NMR-Spektren.

\section{Literatur}

1 H. Oehme und R. Wustrack, Z. Anorg. Allg. Chem., 552 (1987) 215.

2 R. Wustrack und H. Oehme, J. Organomet. Chem., 352 (1988) 95.

3 A. G. Brook und J. J. Crusciel, Organometallics, 3 (1984) 1817.

4 R. Wustrack und H. Oehme, Z. Chem., 29 (1989) 407.

5 J. Ohshita, Y. Masaola und M. Ishikawa, Organometallics, 10 (1991) 3775.

6 A. G. Brook, P. Chiu, J. McClenaghnan und A. J. Lough, Organometallics, 10 (1991) 3292.

7 D. Bravo-Zhivotovskii, V. Braude, A. Stanger, M. Kapon und Y. Apeloig, Organometallics, 11 (1992) 2326.

8 A. Heine, R. Herbst-Irmer, G. M. Sheldrick und D. Stalke, Inorg. Chem., im Drück.

9 L. S. Bartell, F. B. Clippard und T. L. Boates; Inorg. Chem., 9 (1970) 2436.

10 A. Heine und D. Stalke, Angew. Chem., im Drück.

11 U. Edlund, T. Lejon, T. K. Venkatachalam und E. Buncel, J. Am. Chem. Soc., 107 (1985) 6408.

12 U. Edlund, T. Lejon, P. Pyykkö, T. K. Venkatachalam und E. Buncel, J. Am. Chem. Soc., 109 (1987) 5982.

13 H. D. Flack, Acta Crystallogr. Sect. A 39 (1983) 876.

14 G. M. Sheldrick, Acta Crystallogr., Sect. A 46 (1990) 467.

15 G. M. Sheldrick, SHELXL-92 Programm zur Kristallstrukturverfeinerung, Göttingen 1992. 